\title{
HYDROMAGNETIC WAVES AND THE IONOSPHERE
}

\author{
Margaret G. Kivelson \\ Institute of Geophysics and Planetary Physics and Department of Earth and Space Sciences, UCLA \\ David J. Southwood
}

Institute of Geophysics and Planetary Physics, UCLA, and Department of Physics, Imperial College of Science and Technology

\begin{abstract}
The effect of the ionosphere on the detectability, polarization, structure and decay of low frequency magnetospheric hydromagnetic waves has been a topic of interest for thirty years. There are two classes of signal behavior in the atmosphere and ionosphere, one where the ionosphere has little effect and one where the ionospheric conductivity controls the reflection, the amplitude detected on the ground and rate of signal decay. It has been assumed that the latter type of behavior is pertinent in most practical applications. Recent interest in global modes of the magnetospheric cavity requires a reassessment of the assumption. We investigate one simple case, the hydromagnetic surface wave, and discuss what happens in more complicated modes. We conclude that signals in the vicinity of field line resonance will be strongly coupled to the local ionosphere but far away will not.
\end{abstract}

\section{Introduction}

The reflection of a hydromagnetic wave at a conducting boundary is commonly thought to be well understood. Here we contend that the problem needs reassessment particularly in the light of the new interest in global magnetospheric modes as a source of large scale geomagnetic pulsations. We explicitly illustrate some of the difficulties with a simple model.

In derivations of models of the magnetospheric hydromagnetic wave spectrum, a hydromagnetic reflection condition is used to set the ionospheric boundary condition. The boundary condition normally used is

$$
\mathbf{b}_{M}=-\hat{z} \times \mu_{0} \Sigma_{p} \mathbf{E}_{M}
$$

where $b_{M}, \mathbf{E}_{M}$ are the horizontal magnetic and electric fields just above the ionosphere, $\Sigma_{\mathrm{p}}$ is the integrated ionospheric Pedersen conductivity and the $z$ axis is vertical. Condition (1) was originally derived for an Alfven wave incident from the magnetosphere [Dungey, 1963; Nishida, 1964; Hughes and Southwood, 1976].

Condition (1) is appropriate only for a signal like an Alfven wave that contains a strong field-aligned current. Where the current flow in the magnetospheric signal is transverse, the condition is not appropriate. The second type of boundary condition has the form

$$
\mathbf{b}_{M}=(i / \omega h) \hat{z} \times \mathbf{E}_{M}
$$

where $h$ is the height of the ionosphere $(\sim 100 \mathrm{~km}), \omega$ is the angular frequency. Taking even a large value for ionospheric Pedersen conductance $(10 \mathrm{~S})$, one finds that the factor multiplying the ionospheric electric field in (2) is larger than

Copyright 1988 by the American Geophysical Union.

Paper number 88GL03441.

0094-8276/88/88GL-03441\$03.00 the multiplying factor in (1) by about two orders of magnitude at $10 \mathrm{mHz}$ frequency. In fact, throughout the ultra low frequency band, condition (2) is closely equivalent to the condition

$$
\mathbf{E}_{\mathbf{M}}=\mathbf{0}
$$

representing perfect reflection at the ionosphere with the field frozen $\left(E_{M}=0\right)$.

\section{Hydromagnetic Wave Reflection}

In a cold uniform plasma there are two wave modes which propagate, the fast mode and the transverse mode. Figure 1 shows the configurations of the respective wave perturbations for waves incident on a flat ionosphere at a height $h$ above the (flat) ground in the presence of a vertical background magnetic field, B. For both waves, the propagation vector is in the plane of the figure. In the fast mode, the current is entirely horizontal. The wave magnetic field lies in the plane illustrated and has a non-vanishing vertical component. In the transverse mode, the wave magnetic field is purely horizontal and the current, which has a non-vanishing vertical component, lies in the plane illustrated.

\section{Magnetosphere}

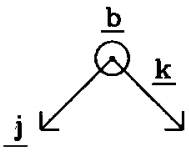

TRANSVERSE MODE

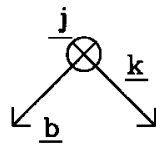

FAST MODE

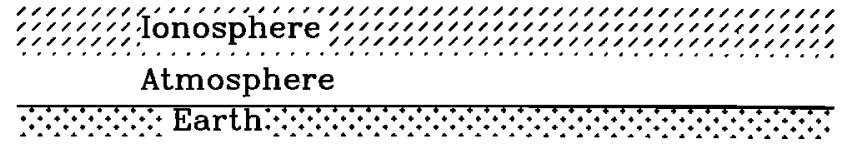

Fig. 1. Schematic of the polarization of the transverse (shear Alfven) wave [left] and the fast mode wave [right] obliquely incident on the ionosphere from the magnetosphere. The plane of the diagram contains $\mathbf{k}$ and the vertical background field, $\mathbf{B}$. For the transverse mode wave, the magnetic perturbation is horizontal (out of the page). For the fast mode wave, the current is horizontal (out of the page).

The different directions of current flow create different responses in the ionosphere. The vertical current in an incident transverse wave drives current into and out of the ionosphere. Below the ionosphere lies the effectively insulating atmosphere in which the vertical current must vanish. Not surprisingly, the 
resistance of the ionosphere governs the reflection of the wave and equation (1) is the immediate consequence. In the fast wave, the current flows horizontally and no current flows between the magnetosphere and the ionosphere. The ionosphere behaves like a thin film that has no effect on the signal. The wave in this case has to match to a free space signal in the atmosphere at the base of which is a perfect conductor (the Earth). Condition (2) follows from integration of Faraday's law [cf. Hughes and Southwood, 1974].

The anisotropy of the ionosphere adds a further complication. The conductivity anisotropy couples the magnetospheric modes. For both types of incident modes, part of the reflected signal emerges in the other mode. For the case of an incident fast mode wave this is unimportant as the small ionospheric electric field drives no significant Hall or Pedersen current. Hall currents driven by an incident transverse mode wave will generate a fast mode signal above the ionosphere and, indeed, the magnetic signal on the ground is proportional to the ionospheric Hall current [Hughes and Southwood, 1974].

Because the signal in the atmosphere from a transverse mode is proportional to the Hall current, the magnetic signal on the ground is rotated through 900 with respect to the magnetic field of the transverse mode in the magnetosphere. For a fast mode wave, the ionosphere has no effect and thus the magnetic field at the ground is aligned in the same sense as the horizontal component of the magnetospheric wave field.

\section{Previous Work}

The two separate classes of behavior identified in this paper have been identified before. In the first work on the topic, Dungey [1958] and Francis and Karplus [1960] derived the result for an incident wave carrying only horizontal current. Dungey [1963] and Nishida [1964, see also 1978] first recognized the existence of the second type of behavior. In Nishida's paper and in Hughes and Southwood's [1976] later treatment, the emphasis was laid on the situation when the incident signal was in the transverse mode. Their reasoning was based on comparison of the dispersion relations of the fast and transverse modes in a uniform plasma. The transverse dispersion relation is

$$
\omega^{2}=\mathrm{k}_{\mathrm{zT}} \mathrm{A}^{2}
$$

where $k_{\mathrm{ZT}}$ is the parallel component of the wave vector and $A$ is the Alfven speed. Let us assume that the incident wave has a horizontal wave number, $\mathrm{kh}$. The component converted into the fast mode on reflection must share the same horizontal variation as the incident wave and also must satisfy the fast mode dispersion relation

$$
\omega^{2}=\left(k_{2 F^{2}}+k_{h}^{2}\right) A^{2}
$$

where $k_{\mathrm{ZF}}$ is the parallel component of the (fast mode) wave vector. For $\mathrm{kh}^{2}>\mathrm{k}_{\mathrm{zT}}{ }^{2}, \mathrm{kzF}_{\mathrm{zF}^{2}}$ must be negative and the reflected fast signal generated during ionospheric reflection of a transverse mode is evanescent in the vertical direction.

The argument that the fast mode can be neglected high in the magnetosphere is not completely general. Long wavelength fast hydromagnetic waves with $k_{h}{ }^{2}<\omega^{2} / A^{2}$ contribute to the ULF wave spectrum in the magnetosphere as do fast mode signals (surface waves) with $\mathrm{kh}^{2}<0$ which are evanescent across the field rather than along it. In both cases, equations (4) and (5) can be satisfied by fast mode waves with real $k_{\mathrm{ZF}}$. This is particularly serious as magnetospheric signals are generally neither pure fast nor pure transverse waves. In such coupled signals new complications arise as our earlier arguments show that standing fast mode signals and standing Alfven wave signals satisfy differing boundary conditions at the feet of magnetic field lines and consequently vary differently with $z$.

\section{Coupled Waves}

In an inhomogeneous plasma, the fast and transverse modes are coupled by the plasma and field inhomogeneities even for a perfectly reflecting ionosphere. Indeed, in the actual magnetosphere it is possible to envisage a pure fast or transverse incident wave only in somewhat exceptional circumstances.

Two types of large scale coupled perturbations are pertinent to the magnetosphere. Field line resonance theory describes the coupling of an inwardly decaying surface wave on the magnetopause to perturbations of a resonant field line within the magnetospheric cavity [Chen and Hasegawa, 1974a; Southwood, 1974]. Global mode theories describe temporally decaying quasi-eigenmodes of the magnetospheric cavity [Kivelson and Southwood, 1985, 1986; Allan et al., 1986, 1987; Inhester, 1987]. The two theories are closely related and are described by the same equations taken in different limits. Surface waves have relatively short perpendicular wavelength; global modes cannot occur when the perpendicular wavelength is small. In both cases, on one or more magnetic shells, field line resonance occurs. Near the resonant field line, the signal resembles the transverse mode. Away from resonance, the signals resemble fast mode waves in most respects, in particular, in containing little vertical current.

The hydromagnetic coupled mode equations for a realistic background field (e.g. dipole) have not yet been solved. Various simpler models have been employed to illustrate the effects of inhomogeneity. Kivelson and Southwood [1987] used the hydromagnetic box model to introduce the idea of global modes. In the model, the field is assumed to be uniform, the field lines are of uniform length and the nonuniformity is introduced by allowing the density (and thus the Alfven velocity) to vary in the $x$ direction.

We use this box model to look at a particularly simple case, the hydromagnetic surface wave [Chen and Hasegawa $1974 \mathrm{~b}]$, where fast and transverse modes are coupled. This wave in important respects resembles a global mode of the system. It occurs where two regions of uniform plasma of different densities are separated by a transition region in which the density varies across the field (see Figure 2). We choose the origin of $x$ to be in the center of the transition region which extends to $x= \pm L$. We take $z$ along the background magnetic field and assume a periodic variation, exp (i $\lambda y)$, in the $y$ direction.

Solutions to the problem are given by various authors [e.g., Chen and Hasegawa, 1974b; Southwood and Hughes, 1983]. All authors have assumed that the variation of the wave along the magnetic field can be represented by the same function of $z$ in all regions (I, II, and III). The implication of our earlier assertions is that this is not so. In regions I and III,

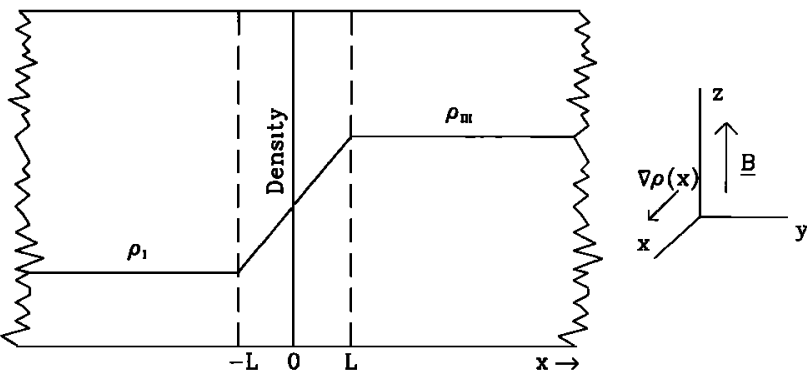

Fig. 2. Density distribution used for the discussion of the hydromagnetic surface wave in a box model of the magnetosphere. The density is uniform except between $x=-L$ and $x=L$. The insert shows the coordinate system, with $z$ parallel to the background field and the density a function of $x$. Periodic structure in $y$ is assumed in the discussion. 
the wave satisfies the fast mode dispersion relation (5). The relevant ionospheric boundary condition is (3). Standing wave structure along the field is given by a $\mathrm{z}$ dependence of the form

$$
\mathrm{e}^{\mathrm{ikz}} \pm \mathrm{e}^{-\mathrm{ikz}}
$$

with real quantized $\mathbf{k}$

$$
k=\mathrm{N} \pi / \boldsymbol{l}
$$

where $\ell$ is the length of the field line and $\mathrm{N}$ is an integer.

In region II, the signal adopts the field line resonance form, the resonant field line occurring at the magnetic shell where the mass density, $\rho_{\text {, equals }}\left(\rho_{\mathrm{I}}+\rho_{\mathrm{III}}\right) / 2\left(=\rho_{\mathrm{O}}\right.$, say). In this region, the boundary condition (1) is appropriate. Standing wave structure along the field again can be written by the form given in equation (6), but $k$ must be complex. The real part $\left(\mathrm{k}_{\mathrm{r}}\right)$ is, once again, $\mathrm{N} \pi /$. The imaginary part is given by

$$
\mathrm{k}_{\mathrm{i}}=\ln \left|\frac{1}{1} \pm \frac{\alpha}{\alpha}\right|
$$

where $\alpha$ is $\mu_{0} \Sigma_{\mathrm{p}} \omega / \mathrm{k}_{\mathrm{r}}$ [Southwood and Hughes, 1983].

The dispersion relation of the surface wave is found by matching the evanescent fast wave solutions in I and III to a field line resonance transverse solution in II. Now with the correct boundary conditions, the z-dependence of the solution in region II is different from that in the other regions. Matching the solutions across the boundaries in $\mathbf{x}$ is more complicated than assumed by earlier authors such as Southwood and Hughes; the functional form along the field cannot be matched by using a single value of $\mathrm{N}$ in equation (7) (Hamieri, personal communication). Matching a signal that has the fundamental structure $(\mathrm{N}=1)$ in region II requires the introduction of $N=1,3,5 \ldots$, components in regions I and III.

Provided the imaginary part of $\mathbf{k}$ in region II is small, matching may still be approximately achieved with a single value of $\mathrm{N}$ and the principal features of the solution remain unchanged. The frequency is complex. The real part is given by $\omega=k_{r} B /\left(\mu_{o} \rho_{o}\right) 1 / 2$. The damping decrement can be attributed to two causes. Energy is lost into the ionosphere, but the dominant process (phase mixing damping) is that energy is fed into oscillations in a narrow field line resonance region surrounding $x=0$ [see Southwood and Hughes, 1983].

The use of the correct boundary conditions has minor implications for the energy deposition from the wave. If the only source of dissipation is in the ionosphere, energy may leave the system only through that part of the boundary where less than perfect ionospheric reflection occurs, i.e. in region II, and it follows that the energy dissipation in the wave is more localized than in previous predictions. However, as the dissipative condition holds good in the region where field line resonance occurs and the surface wave itself damps by feeding energy into the resonance location, the bulk of the energy deposition would be there in any case.

In magnetospheric applications, the thin transition region is identified as the plasmapause [Chen and Hasegawa, 1974b], and has an ionospheric footprint highly localized in latitude. The region where the transverse mode signal is dominant is thin and the signal associated with it may not be easily detected on the ground [see Hughes and Southwood, 1976]. Outside the transition region the magnetospheric signal is in the fast mode (but evanescent); the ionosphere has little effect on the signal and the magnetic signal recorded at the ground is similar to that recorded just above the ionosphere in the magnetosphere.

\section{Global Modes}

For the hydromagnetic surface wave described in the previous section, the analysis of boundary conditions simplifies because one type of boundary condition is applied in regions I and III and the other in region II where the field line resonance occurs. The situation is less clear cut in the case of the global mode waves introduced by Kivelson and Southwood [1985]. Computations of global mode structure have been done by several authors [e.g. Allan et al., 1986; Zhu and Kivelson, 1988]. Global mode solutions in the hydromagnetic box model are effectively the eigenmodes of the fast mode and have a standing structure both across and along the field.

The distinction between the surface wave situation and the global mode is that the plasma parameters are assumed to vary smoothly and there is no sharp transition in the wave structure from fast mode like characteristics to transverse characteristics and thus no clear rule for using boundary condition (1) or condition (3). Allan et al. [1986] used condition (1), whereas Zhu and Kivelson [1988] assumed a perfectly reflecting ionosphere as in condition (3).

In the uniform case the critical distinction between boundary conditions (1) and (3) is dependent on the presence or absence of parallel current in the magnetospheric wave.

In the global mode, the field aligned current peaks strongly at the resonance but there is some field aligned current present everywhere. As we intend to show in more detail elsewhere, at any given location the global mode signal is a true mixture of fast and transverse modes. The component of the signal directly associated with field aligned currents and their horizontal closure currents peaks in amplitude at the field line resonance location and decays on either side. The residual component in which current flows entirely horizontally is largest far from the resonance. Although both boundary conditions need to be applied to different parts of the signal at each point, near resonance the part obeying the "Alfven wave" reflection condition, equation (1), is dominant. Far away, the other part dominates and condition (2) needs to be used.

There are various physical consequences of the results described here. Condition (1) is a dissipative boundary condition; condition (2) is not. Thus, our results suggest that energy deposition is even more effectively confined to the resonance region than hitherto believed.

Predicting the polarization seen on the ground is also subtle. Near resonance the ionospheric currents strongly modify the polarization between magnetosphere and ground. Hall currents flowing perpendicular to the dissipative Pedersen currents set up a magnetic perturbation below the ionosphere at right angles to the magnetospheric magnetic perturbation. Away from resonance, the signal is predominantly in the fast mode and the magnetospheric signal penetrates to the ground. Thus, polarization rotation becomes less and less important as one moves away from the resonance.

For a qualitative description, one may be guided by the fact that the magnetic signal on the ground always is oriented such that the vertical current in the atmosphere is small or, equivalently, that the horizontal signal is always magnetostatic and derivable from a potential. Near resonance that potential is proportional to the Hall conductivity of the local ionosphere; far away where the magnetospheric signal can come through to the ground, it is not. This point was made also by Hughes [1974] who remarked that the wave polarization seen on the ground is independent of the polarization of the magnetospheric fields.

Here we have limited the discussion to a vertical field geometry but the arguments also apply if the background field is inclined. We expect to treat the extension of our arguments to the inclined field and to consider the problems raised in this paper in a more mathematically detailed manner in a subsequent study.

Acknowledgements. The authors thank Xiaoming Zhu and Eli Hamieri for useful discussions and John W. Belcher and the Center for Space Research at M.I.T. for hospitality during the completion of the work. This work was supported 
by the Division of Atmospheric Sciences of the National Science Foundation under grant ATM 86-10858.

\section{References}

Allan, W., S. P. White, and E. M. Poulter, Impulse-excited hydromagnetic cavity and field line resonances in the magnetosphere, Planet. Space Sci., 34, 371, 1986.

Allan, W., E. M. Poulter, and S. P. White, Structure of magnetospheric MHD resonances for moderate "azimuthal" asymmetry, Planet. Space Sci.,in press, 1987.

Chen, L. and A. Hasegawa, A theory of long period magnetic pulsations 1 . Steady state excitation of field line resonances, J. Geophys. Res., 79, 1024-1032, 1974a.

Chen, L. and A. Hasegawa, A theory of long period magnetic pulsations 2. Impulse excitations of surface eigenmode, J. Geophys. Res., 79, 1033-1037, $1974 \mathrm{~b}$.

Dungey, J. W., Cosmic Electrodynamics, Cambridge University Press, Cambridge, 1958.

Dungey, J. W., Hydromagnetic waves and the ionosphere, in Proceedings of the International Conference on the Ionosphere, p.230, 1963.

Francis, W. E., and R. Karplus, Hydromagnetic waves in the ionosphere, J. Geophys. Res., 65, 3593, 1960.

Hughes, W. J., The effect of the atmosphere and ionosphere on long period magnetospheric micropulsations, Planet. Space Sci., 22, 1157, 1974.

Hughes, W. J. and D. J. Southwood, The effect of the atmosphere and ionosphere on magnetospheric micropulsation signals, Nature, 249, 493, 1974.

Hughes, W. J. and D. J. Southwood, The screening of micropulsation signals by the atmosphere and ionosphere, J. Geophys. Res., 81, 3340, 1976.
Inhester, B., Numerical modelling of hydromagnetic wave structure in the magnetosphere, J. Geophys. Res., 92 , 4571, 1987.

Kivelson, M. G., and D. J. Southwood, Resonant ULF waves: A new interpretation, Geophys, Res. Lett., 12, 49, 1985.

Kivelson, M. G. and D. J. Southwood, Coupling of global magnetospheric MHD eigenmodes to field line resonances, J. Geophys. Res., 91, 4345, 1986.

Nishida, A., Ionospheric screening effect and storm sudden commencement, J. Geophys. Res., 69, 1861, 1964.

Nishida, A. Geomagnetic Diagnosis of the Magnetosphere, Springer-Verlag, New York, 1978.

Southwood, D. J., Some features of field line resonance in the magnetosphere, Planet. Space Sci., 22, 483-491, 1974.

Southwood, D. J. and W. J. Hughes, Theory of hydromagnetic waves in the magnetosphere, Space Science Rev., 35, 301, 1983.

Zhu, X., and M. G. Kivelson, Analytic formulation and quantitative solutions of coupled ULF wave problem, $\mathbf{J}$. Geophys. Res., 93, 8602, 1988.

UCLA Institute of Geophysics and Planetary Physics Publication Number 3097

M. G. Kivelson and D.J. Southwood, Institute of Geophysics and Planetary Physics, University of California, Los Angeles, CA 90024-1567

(Received: May 12, 1988; revised: August 1, 1988; accepted: August 8,1988 .) 